188 Nicolina Silvana Romano-Lieber/Marcelo Ferreira Carlos Cunha/Eliane Ribeiro

\title{
A Farmácia como Estabelecimento de Saúde
}

\author{
PHARMACY AS A HEALTH ESTABLISHMENT
}

\author{
Nicolina Silvana Romano-Lieber $\left.{ }^{*}\right)$ \\ Marcelo Ferreira Carlos Cunha ${ }^{(* *)}$ \\ Eliane Ribeiro ${ }^{(* * *)}$
}

\section{INTRODUÇÃO}

Farmácias e drogarias são, desde longa data, marcos representativos na constituição das urbes. Sua posição singular entre "estabelecimento comercial" e "órgão da saúde" vem promovendo a necessidade de regulamentações específicas e, não raro, também de conflitos de interesse dos mais diversos.

${ }^{*}$ *) Farmacêutica-Bioquímica. Professora Doutora do Departamento de Prática de Saúde Pública da Faculdade de Saúde Pública da Universidade de São Paulo. Especialista, mestre e doutora em Saúde Pública. E-mail: <nicolina@usp.br>.

$\left.{ }^{(*}\right)$ Farmacêutico-Bioquímico. Mestre em Saúde Pública pela Faculdade de Saúde Pública da Universidade de São Paulo.

$\left.{ }^{(* \star *}\right)$ Farmacêutica-Bioquímica. Professora Doutora do Departamento de Farmácia da Faculdade de Ciências Farmacêuticas da Universidade de São Paulo. Diretora do Serviço de Farmácia do Hospital Universitário da Universidade de São Paulo. Mestre em Administração de Empresas pela Fundação Getúlio Vargas e doutora em Ciências Farmacêuticas pela Universidade de São Paulo. Recebido em 05.10.08. Reapresentado em 13.10.08. Aprovado em 15.10.08. 
No Brasil, a normalização das atividades nesses estabelecimentos teve um marco importante em 1973, com a publicação da Lei Federal n. 5.991, a qual dispõe sobre o controle sanitário do comércio de drogas, medicamentos, insumos farmacêuticos e correlatos. Desde então, têm havido adequações da legislação pertinente com vistas a atender as transformações ocorridas nessa área, bem como as expectativas da população. Neste sentido, foi particularmente importante a instituição da Política Nacional de Assistência Farmacêutica, formalizada pela Resolução n. 338, de 6.5.04, do ConseIho Nacional de Saúde.

A Assistência Farmacêutica, enquanto uma das atribuições previstas também para as farmácias e drogarias, define-se como um conjunto de ações voltadas à promoção, proteção e recuperação da saúde, tanto individual, como coletiva, onde o medicamento é entendido como um insumo essencial. Dentre este conjunto de ações, destaca-se o acompanhamento e a avaliação do uso de medicamentos, conforme uma perspectiva de obtenção de resultados concretos e de melhoria da qualidade de vida da população.

Em contraposição a essa crescente convergência de entendimento das farmácias e drogarias como um serviço especializado do setor saúde, à disposição do público, o legislativo do Estado de São Paulo aprovou o Projeto de Lei n. 955, de 2003, proposto pela deputada Ana do Carmo (PT), disciplinando o comércio de artigos de conveniência em farmácias e drogarias. Embora objeto de Veto Total do então governador Geraldo Alckmin, o Projeto foi convertido na Lei n. 12.623, em 25 de junho de 2007, dando origem, então, à Ação Direta de Inconstitucionalidade proposta pelo atual governador José Serra ao Supremo Tribunal Federal.

O propósito desse comentário é examinar o sentido desta Ação não apenas à luz da evolução da Legislação Federal desde 1973, mas também considerando o papel precípuo das farmácias e drogarias enquanto estabelecimentos de saúde que lidam com um produto singular, o medicamento. Medicamentos decorrem da evolução do conhecimento científico. O seu uso, derivado da prescrição e da comercialização, também pode colocar em risco a saúde das populações. Portanto, as formas de controle desse risco e as recomendações supranacionais para tal fim não podem deixar de ser consideradas nessa análise.

\section{ARCABOUÇO LEGAL E NORMATIVO}

A Lei n. 5.991, de 17.12.73, diploma legal regulamentado pelo Decreto n. 74.170/74, define que o comércio de drogas, medicamentos e de insumos farmacêuticos é privativo das empresas e dos estabelecimentos nela definidos. Entretanto, observa que poderá ser extensivo às farmácias e drogarias o comércio de outros produtos, tais como, aparelhos e acessórios, produtos 
utilizados para fins diagnósticos e analíticos, odontológicos, veterinários, de higiene pessoal ou de ambiente, cosméticos e perfumes, exercido por estabelecimentos especializados, desde que observado o disposto em lei federal e na supletiva dos Estados, do Distrito Federal e dos Territórios.

As normas suplementares, editadas desde então, seguem estritamente os limites determinados na Lei Federal n. 5.991/73, com respeito às categorias de produtos a serem comercializados nos estabelecimentos farmacêuticos.

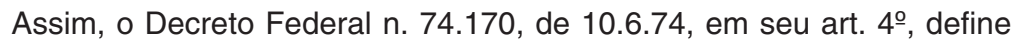
que:

É permitido às farmácias e drogarias exercerem o comércio de determinados correlatos, como aparelhos e acessórios usados para fins terapêuticos ou de correção estética, produtos utilizados para fins diagnósticos e analíticos, de higiene pessoal ou de ambiente, o de cosméticos e perfumes, os dietéticos mencionados no parágrafo único in fine do artigo anterior, os produtos óticos, de acústica médica, odontológicos, veterinários e outros, desde que observada a legislação especifica federal e a supletiva, pertinente, dos Estados, do Distrito Federal e dos Territórios.

A Lei Federal n. 6.360, de 23.9.76 amplia a lista quando inclui “... saneantes domissanitários, produtos destinados à correção estética e outros adiante definidos".

Mais recentemente, com vistas a disciplinar o comércio desses outros produtos, a Resolução RDC n. 173, de 8.7.03, que altera a Resolução n. 328, de 22.7.99, da Agência Nacional de Vigilância Sanitária (ANVISA) determina que é vedado à farmácia e drogaria.

Expor a venda produtos alheios aos conceitos de medicamento, cosmético, produto para saúde e acessórios, alimento para fins especiais, alimento com alegação de propriedade funcional e alimento com alegação de propriedades de saúde.

Determina, ainda, que os alimentos acima referidos somente podem ser vendidos em farmácias quando possuírem forma farmacêutica e estiverem devidamente legalizados no órgão sanitário competente e apresentarem Padrão de Identidade e Qualidade (PIQ) estabelecido em legislação específica.

Além destas normas supletivas, merecem destaque outros dois esforços para regular, em complemento à Lei n. 5.991/73, os produtos que devem ser comercializados nos estabelecimentos farmacêuticos.

Em fevereiro de 2006, o Conselho Regional de Farmácia do Estado de São Paulo (CRF-SP), tendo como base a Lei n. 5.991/73 e as normas suplementares citadas, publica uma relação de produtos alheios ao ramo farmacêutico. 
$\mathrm{Na}$ relação, observam-se alimentos comuns, artigos de uso pessoal, artigos em geral, incluindo cine foto, vídeo e equipamentos eletroeletrônicos, produtos esotéricos e religiosos, produtos químicos, agrícolas e veterinários.

Da mesma forma, a Consulta Pública n. 69/07 da ANVISA, apresenta uma relação de produtos permitidos e outra de produtos vedados ao ramo. Nesta última, inclui as categorias: alimentos comuns, artigos de uso doméstico e pessoal, artigos de tabacaria, materiais de cine, foto e vídeo e produtos saneantes e veterinários.

Cabe ressaltar que existe regulamentação para a venda desses produtos. Assim, a Lei Federal n. 9.069, de 29 de junho de 1995, conceituou a loja de conveniência e drugstore como estabelecimentos que mediante autoserviço ou não, comercializam diversas mercadorias. Entre estas, é dada ênfase àquelas de primeira necessidade, dentre as quais alimentos em geral, produtos de higiene e apetrechos domésticos, podendo funcionar em qualquer período do dia e da noite, inclusive domingos e feriados, prevendo, nesses casos, que o estabelecimento não dependerá de assistência técnica ou responsabilidade profissional. A maioria dos produtos considerados de conveniência não se enquadra na classificação de produtos correlatos a drogas, medicamentos e insumos farmacêuticos.

\section{FARMÁCIA COMO ESTABELECIMENTO DE SAÚDE}

A Lei n. 5.991/73 caracteriza farmácias e drogarias como estabelecimentos de saúde, cuja atuação se situa, exclusivamente, nos domínios da defesa e proteção da saúde individual ou coletiva. Isto está explicitado em seu art. 55 em que dispõe que é proibida a utilização de "qualquer dependência da farmácia ou drogaria como consultório, ou outro fim diverso do licenciamento", qual seja, a autorização para comercializar drogas, medicamentos, insumos farmacêuticos e correlatos concedida pela autoridade sanitária estadual ou distrital.

No Brasil, as farmácias e drogarias ainda estão distanciadas do seu papel sanitário; a dispensação de medicamentos nem sempre é entendida como processo de assistência à saúde; há insuficiência de orientação farmacêutica no momento da dispensação de medicamentos, tanto em estabelecimentos privados como nos públicos; e o profissional farmacêutico poucas vezes está presente nas farmácias para prestar adequadas informações e orientações. Neste sentido, as leis suplementares à Lei n. 5.991/73 foram concebidas na reorientação dos estabelecimentos farmacêuticos como estabelecimentos de saúde.

O papel das farmácias e drogarias vem sendo discutido à luz de uma nova orientação. Em 1996, a Organização Mundial de Saúde (OMS) estabeleceu que "a missão da prática farmacêutica é prover medicamentos e outros 
produtos e serviços e auxiliar as pessoas e a sociedade a utilizá-los da melhor forma possível" (1). Posteriormente, em 2006, a mesma OMS publicou um manual como referência para a reorientação da educação e da prática farmacêutica e a incorporação da atenção farmacêutica nesta prática ${ }^{(2)}$.

No Brasil, a Política de Medicamentos(3) a Política Nacional de Assistência Farmacêutica, já citada, além da $1^{\text {a }}$ Conferência Nacional de Vigilância Sanitária (2001) e a 1aㅡ Conferência Nacional sobre Medicamentos e Assistência Farmacêutica (2003), convergem para a perspectiva de um novo modelo de farmácia que defina as suas funções no âmbito da recuperação, proteção e promoção da saúde.

A 1aㅡ Conferência Nacional sobre Medicamentos e Assistência Farmacêutica vai além, com uma proposta de "transformar o conceito de estabelecimentos farmacêuticos privados em estabelecimentos de serviços de saúde, integrados ao SUS de forma complementar, conforme legislação vigente"(4).

A observância desses preceitos caracteriza as farmácias e drogarias como estabelecimentos de saúde que assistem ao cliente, por meio da prestação da Assistência Farmacêutica e comercialização de produtos relacionados à saúde.

A compreensão desse conceito é de suma importância para o desenvolvimento da Assistência Farmacêutica na rede privada e pública do país acesso aos produtos e a um conjunto de ações de atenção à saúde, com serviços qualificados, incluindo benefício social, atendimento com foco na saúde do paciente e não somente no lucro comercial; a farmacovigilância e a Atenção Farmacêutica que, por sua vez, envolvem a informação ao cliente, por meio do seguimento da sua farmacoterapia e a educação sanitária, entre outras.

Entende-se que transformar esses estabelecimentos em meros distribuidores de produtos, incluindo os medicamentos, descaracteriza sua função social na área da saúde, possibilitando o entendimento do medicamento como simples produto de consumo, que não oferece risco à população e que

(1) ORGANIZAÇÃO PAN-AMERICANA DA SAÚDE. ORGANIZAÇÃO MUNDIAL DA SAÚDE. CONSELHO FEDERAL DE FARMÁCIA. O papel do farmacêutico no sistema de atenção à saúde. Brasília: Organização Mundial de Saúde/Organização Pan-Americana da Saúde/Conselho Federal de Farmácia, 2004. p. 83. Disponível em: <http://www.opas.org.br/medicamentos/site/UploadArq/ HSE_PFS_BPF_1004.pdf>. Acesso em: 12 set. 2008.

(2) WORLD HEALTH ORGANIZATION. INTERNATIONAL PHARMACEUTICAL FEDERATION. Developing pharmacy practice: a focus on patient care, handbook 2006. Netherlands: World Health Organization/International Pharmaceutical Federation. 2006. p. ix. Disponível em: <http:// www.correofarmaceutico.com/documentos/041206WHO_PSM_PAR_2006.5.pdf>. Acesso em: 10 set. 2008.

(3) Aprovada pela Portaria n. 3916, de 30 de outubro de 1998.

(4) MINISTÉRIO DA SAÚDE. CONSELHO NACIONAL DE SAÚDE. 1 Conferência Nacional de Medicamentos e Assistência Farmacêutica. Relatório Final. 2005. p. 86. Disponível em: <http:// dtr2001.saude.gov.br/editora/produtos/livros/pdf/05_0069_M.pdf>. Acesso em: 10 set. 2008. 
não requer condições especiais de armazenamento sendo, sua comercialização, por conseguinte, permitida em qualquer local.

\section{RISCOS RELACIONADOS AO USO DE MEDICAMENTOS}

O desenvolvimento da indústria farmacêutica, a partir da década de 40, faz com que medicamentos mais potentes, mas também mais tóxicos, sejam colocados no mercado. Muitas doenças, principalmente as infecciosas são, agora, curadas. Entretanto, não sem risco à população devido ao seu uso indiscriminado. $O$ fracasso da terapêutica medicamentosa, seja por ineficácia ou por eventos adversos, ocasiona perdas em vidas e em recursos econômicos.

A mortalidade e morbidade relacionadas a medicamentos se tornaram um problema de saúde pública. Os medicamentos apresentam uma série de características distintas dos antigos fármacos manipulados. Sua indicação é cada vez mais específica e seu potencial cada vez maior; as reações adversas são mais graves; as posologias mais complexas e os riscos de interação mais freqüentes. Além disso, são mais caros e o perfil dos pacientes que os estão recebendo modificou-se. A população mundial envelheceu, alterando a prevalência e incidência de doenças e a relação de necessidades socioeconômicas.

Revisão da literatura apontou as reações adversas a medicamentos entre a 4 $4^{\underline{a}}$ e a 6 ${ }^{\underline{a}}$ causa de morte nos Estados Unidos, sendo que a incidência de reações adversas fatais foi de $0,23 \%$ a $0,41 \%{ }^{(5)}$. Para o sistema de saúde americano, os custos com morbidade e mortalidade relacionadas a medicamentos foram estimados em mais de US\$ 76,6 bilhões $^{(6)}$.

Estudo realizado na Itália mostrou que dos 2.442 casos assistidos na Emergência do Hospital, 1,3\% estavam relacionados a medicamentos. Dos 480 pacientes hospitalizados, 3,6\% (17) foram em virtude de eventos adversos, corroborando a evidência de que as doenças relacionadas ao uso dos medicamentos são um problema importante de saúde pública ${ }^{(7)}$.

Na França, no período de 1998 a 2004, 21 novos fármacos foram retirados do mercado por razões de segurança ${ }^{(8)}$.

(5) LAZAROU Jason; POMERANZ, Bruce H.; COREY, Paul. Incidence of adverse drug reactions in hospitalized patients - a meta-analysis of prospective studies. Journal of the American Medical Association, Chicago, v.279, n.15, p. 1200-1205, 1998.

(6) JOHNSON Jeffrey A.; BOOTMAN Lyle J. Drug-related morbidity and mortality: a cost-of-illness model. Archives of Internal Medicine, Chicago, v. 155, n. 18, p. 1949-1956, 1995.

(7) CAPUANO Annalisa; MOTOLA Giulia; RUSSO Francesco; AVOLIO Amalia; FILIPPELLI Amelia; ROSSI Francesco; MAZZEO Filomena. Adverse drug events in two emergency departments in Naples, Italy: an observational study. Pharmacology Research, Londres, v. 50, n. 6, p. 631-636, 2004.

(8) OLIVER Pascale; MONTASTRUC Jean-Louis. The nature of the scientific evidence leading to drug withdrawals for pharmacovigilance reasons in France. Pharmacoepidemiology and drug safety, Chichester, v. 15, n. 11, p. 808-812, 2006. 
Mesmo os produtos considerados mais seguros provocam eventos adversos graves. No período de abril de 2002 a março de 2007, o Instituto Nacional de Saúde Italiano, recebeu 233 notificações espontâneas de suspeitas de eventos adversos causados por produtos registrados como naturais e para a saúde (preparações contendo própolis e homeopáticas). A maioria das suspeitas foram eventos graves. Trinta e cinco por cento ocasionaram hospitalização, colocando $6 \%$ em risco de morte e provocando 2 óbitos ${ }^{(9)}$. No Brasil, os medicamentos se apresentam como o principal agente tóxico, tendo respondido por aproximadamente $30 \%$ dos casos de intoxicação humana registrados pelo Sistema Nacional de Informações Tóxico-Farmacológicas do Ministério da Saúde em 2006 ${ }^{(10)}$.

Além desse quadro, no Brasil, a dispensação de medicamentos freqüentemente não é entendida como processo de atenção à saúde. Encontram-se enraizadas a cultura e a prática da automedicação, tendo o medicamento como centro do cuidado da saúde. Predomina a noção de que para o bom atendimento à saúde são necessárias prescrições de medicamentos e solicitação de exames complementares. Os usuários conhecem insuficientemente seus direitos à saúde.

\section{AÇÕES DE SAÚDE DO FARMACÊUTICO EM FARMÁCIAS E DROGARIAS}

Tendo em vista o quadro apresentado, não é sem razão que a ANVISA tenha aberto Consulta Pública para consolidar um Regulamento Técnico de Boas Práticas Farmacêuticas em Farmácias e Drogarias, a já citada CP n. 69/07. $\mathrm{O}$ documento estabelece que, além da dispensação, seja permitida às farmácias e drogarias a prestação de alguns serviços farmacêuticos. De forma complementar à CP n. 69/07, o Conselho Regional de Farmácia do Estado de São Paulo sugere algumas ações para redução de riscos relativos a medicamentos, buscando fazer com que esses estabelecimentos funcionem de forma mais integrada ao sistema de saúde. Assim, propõe-se, entre outros, o acompanhamento farmacoterapêutico; a aplicação subcutânea, intramuscular, intradérmica e endovenosa de medicamentos injetáveis; serviços de inalação e/ou nebulização; monitoramento da pressão arterial; medição da temperatura corporal; monitoramento da glicemia capilar e a realização de curativos de pequeno porte.

(9) MENNITI-IPPOLITO Francesca; MAZZANTI Gabriela; SANTUCCIO Carmela; MORO Paola A; CALAPAI, Gioacchino; FIRENZUOLI, Fabio; VALERI, Andrea; RASCHETTI, Roberto. Surveillance of suspected adverse reactions to natural health products in Italy. Pharmacoepidemiology and drug safety, Chichester, v. 17, n. 6, p. 626-635, 2008.

(10) BRASIL. MINISTÉRIO DA SAÚDE. FUNDAÇÃO OSWALDO CRUZ. Sistema Nacional de Informações Tóxico-Farmacológicas - SINITOX. Disponível em: <http://www.fiocruz.br/sinitox/2006/ tab4_brasil.pdf> Acesso em: 10 set. 2008. 
Para estimular o desenvolvimento de ações de saúde em farmácias e drogarias, outras iniciativas já têm sido implementadas, como o Programa de Farmácias Notificadoras, lançado pela ANVISA em parceria com o Centro de Vigilância Sanitária e o Conselho Regional de Farmácia de cada Estado, com o objetivo de ampliar as fontes de notificação de casos suspeitos de efeitos adversos a medicamentos e de queixas técnicas de medicamentos.

Há também o Projeto de Fracionamento de Medicamentos, de responsabilidade do farmacêutico e que deve ser realizado de acordo com Boas Práticas Farmacêuticas. O fracionamento é um instrumento para a promoção do uso racional de medicamentos, pois permite disponibilizar o produto na quantidade adequada e suficiente para o tratamento, evitando que haja sobras de medicamentos em casa, diminuindo a possibilidade da automedicação e, conseqüentemente, de eventos adversos advindos de seu uso.

Alguns Estados contam ainda com farmácias e drogarias envolvidas em Campanhas de Educação em Saúde, mobilizando profissionais e estabelecimentos na orientação dos usuários sobre sintomas e fatores de risco e prevenção de algumas doenças. Além disso, propõe-se que os estabelecimentos participem do programa de coleta de medicamentos a serem descartados pela comunidade, com vistas a preservar a saúde pública e a qualidade do meio ambiente, considerando os princípios da biossegurança de emprego de medidas técnicas, administrativas e normativas para prevenir acidentes, preservando a saúde pública e o meio ambiente.

A perspectiva do farmacêutico como profissional de saúde, com ênfase no usuário e com responsabilidades em relação ao processo de utilização dos medicamentos e seus resultados, ao invés de um mero distribuidor de medicamentos, vem sendo discutida pela Organização Mundial de Saúde e outras organizações internacionais desde 1988, quando houve uma reunião em Nova Delhi e, posteriormente, em 1993, em Tóquio(11). Essas reuniões foram seguidas pela adoção da resolução da 47aㅡ Assembléia Mundial de Saúde (WHA 47.12)(12) sobre o papel do farmacêutico, em maio de 1994, em apoio à estratégia revisada da OMS sobre medicamentos. Nestas, apontaram-se princípios que orientavam o papel do farmacêutico no sistema de atenção à saúde.

A partir dessas reuniões, em 1996, a OMS divulgou documento que trata das Boas Práticas em Farmácia, em ambientes comunitários e hospita-

(11) MARIN, Nelly; LUIZA, Vera Lúcia; OSORIO-DE-CASTRO, Claudia G. Serpa; MACHADO-DOSSANTOS, Silvio (Orgs.) Assistência farmacêutica para gerentes municipais. Rio de Janeiro: OPAS/ OMS, 2003. 373 p. Disponível em: <http://www.opas.org.br/medicamentos/site/UploadArq/0080.pdf>. Acesso em: 17 set. 2008.

(12) WHA47.12 Role of the pharmacist in support of the who revised drug strategy. Pharmaceutical Care España, Barcelona, v. 2, n. 4, p. 282-284, 2000. Disponível em: <http://www.pharmaceutical-care.org/ contenido.php? mod=archivobusqueda\&menu=3.2\&id=221\&tipo=completo>. Acesso em: 12 set. 2008. 
lares $^{(13)}$. Esse documento estabelece como principais elementos das Boas Práticas em Farmácia: a) atividades associadas à promoção da saúde e prevenção de enfermidades e alcance de objetivos sanitários; b) atividades associadas ao abastecimento e uso de medicamentos e itens para a administração de medicamentos ou de alguma forma relacionados com o tratamento; c) atividades associadas ao autocuidado, incluindo orientação sobre um medicamento e, quando apropriado, dispensação de medicamento ou outro tratamento para os sintomas de enfermidades que podem ser devidamente tratadas pelo próprio usuário; d) atividades associadas à influência na prescrição e no uso de medicamentos. Certamente, essas atividades vão além da prática do profissional farmacêutico, caracterizando um campo de atuação de natureza multiprofissional e interdisciplinar.

Segundo a OMS ${ }^{(14)}$, entre as intervenções que podem ser aplicadas com o objetivo de utilizar os medicamentos de forma racional, estão também a regulamentação e fiscalização apropriadas. Estas, por sua vez, incluem o registro de profissionais de saúde - médicos, enfermeiras e demais profissionais - assegurando que tenham a necessária competência para a prática relacionada com diagnóstico, prescrição e dispensação. O licenciamento de estabelecimentos farmacêuticos, como farmácias e distribuidoras, deve assegurar o cumprimento dos padrões de funcionamento e de dispensação.

Estas diretrizes foram seguidas por muitos países. Por exemplo, na Espanha, a dispensação é uma das atividades da farmácia, representando um dos processos da cadeia terapêutica, devendo, portanto, ser entendida e praticada como ato assistencial e como garantia do processo global de uso do medicamento. Desta forma, a presença e atuação do farmacêutico são requisitos inescusáveis para a dispensação de medicamentos. Este profissional deve prestar serviço básico à população por meio de informações sobre o uso dos medicamentos, praticando seguimento da terapia farmacológica do paciente, educação sanitária etc. O não cumprimento destas atividades pode ser caracterizado como um ato de negligência do profissional ${ }^{(15)}$.

Em 2006, durante o VII Congresso Nacional da Rede Unida, uma oficina de trabalho foi realizada, para discutir o uso racional de medicamentos na perspectiva multiprofissional e propor estratégias para potencializar as ações nesse campo. Dentre estas, estão: a) promover a educação permanente dos atores envolvidos; b) considerar a farmácia e drogaria como estabelecimen-

(13) ORGANIZAÇÃO PAN-AMERICANA DA SAÚDE. ORGANIZAÇÃO MUNDIAL DA SAÚDE. CONSELHO FEDERAL DE FARMÁCIA. O papel do farmacêutico no sistema de atenção à saúde, cit., p. 85-86. (14) WORLD HEALTH ORGANIZATION. Promoting rational use of medicines: core components. WHO Policy Perspectives on Medicines. n. 5; Sept. 2002. p. 5. Disponível em: <http://www.who.int/ medicines/publications/policyperspectives/ppm05en.pdf>. Acesso em: 10 set. 2008.

(15) HERRERA CARRANZA, Joaquín. Aspectos éticos de la Atención Farmacéutica desde la perspectiva de la cadena terapéutica del medicamento. Pharmaceutical Care España, Barcelona, v. 3, n. 3, p. 223-226, 2001. 
tos de saúde; e c) proibir a comercialização de artigos alheios ao ambiente sanitário. Em relação às políticas e diretrizes, foi proposta a revisão da legislação, de forma a melhor caracterizar as farmácias e drogarias como estabelecimentos de saúde. A responsabilidade por esta revisão deveria ser assumida pelo Ministério da Saúde, ANVISA, em conjunto com o Legislativo e entidades profissionais e estudantis ${ }^{(16)}$.

O entendimento das farmácias e drogarias como estabelecimentos de saúde é relevante para o desenvolvimento da Assistência Farmacêutica na rede privada e pública do país, cuja formulação enfatiza o atendimento dirigido à saúde do paciente e não apenas ao lucro comercial.

\section{CONSIDERAÇÕES FINAIS}

O exposto mostra que, dentro de uma perspectiva de promoção da saúde, a iniciativa do atual governador procede. À parte das considerações constitucionais, a Lei Estadual n. 12.623/07 contrapõe-se ao conjunto de Leis Federais, cuja evolução vem buscando trazer conotação mais precisa ao significado restrito de estabelecimento de saúde às farmácias e drogarias, em consonância com as recomendações supranacionais que o país procura atender.

A Ação Direta de Inconstitucionalidade, em face da Lei Estadual n. $12.623 / 07$, orienta-se no fato de que, na sua concepção, existe contrariedade com a competência da União em legislar sobre as normas gerais da proteção e defesa da saúde. A lei extrapola os limites determinados pela Lei Federal que disciplina a matéria ao estabelecer a venda de produtos de conveniência em farmácias e drogarias. Em resumo, como salientado na própria Ação, o legislador estadual, ao disciplinar o comércio de artigos de conveniência em farmácias e drogarias, não se restringiu àqueles produtos que se enquadrassem na modalidade de correlatos, conforme a Lei n. 5.991/ 73, diretamente ligados à defesa e proteção da saúde individual ou coletiva, mas entendeu como lícita a comercialização de todo e qualquer artigo de conveniência, contemplando inúmeros itens que nada têm a ver com a saúde pública.

É mundial e crescente a tendência em conceber farmácias e drogarias como estabelecimentos onde é possível realizar várias ações de saúde, em complemento e integradas ao sistema de saúde. Entende-se que transfor-

(16) ORGANIZAÇÃO PAN-AMERICANA DA SAÚDE. Oficial de trabalho uso racional de medicamentos na perspectiva multiprofissional/Organização Pan-Americana da Saúde; Brasil. Ministério da Saúde; Agência Nacional de Vigilância Sanitária. — Brasília: Organização Pan-Americana da Saúde, 2007. p. 25. Disponível em: <http://portal.saude.gov.br/portal/arquivos/pdf/urm_rede_unida.pdf>. Acesso em: 12 set. 2008. 
mar estes estabelecimentos em meros distribuidores de produtos, incluindo os medicamentos, descaracteriza a sua função social na área da saúde, como, também, possibilita o entendimento do medicamento como um simples produto de consumo, que não oferece risco à população, ou mesmo, que dispense condições especiais de armazenamento, traduzindo, por conseguinte, a liberdade de sua comercialização em qualquer local.

A Ação Direta de Inconstitucionalidade permitirá resgatar o esforço de transformação das farmácias e drogarias em estabelecimentos de saúde à luz dos avanços da ciência nessa área, é coerente com o reposicionamento conceitual dos órgãos supranacionais e contribuirá para dar forma a um sistema de saúde mais eficiente.

\section{REFERÊNCIAS BIBLIOGRÁFICAS}

BRASIL. MINISTÉRIO DA SAÚDE. FUNDAÇÃO OSWALDO CRUZ. Sistema Nacional de Informações Tóxico-Farmacológicas - SINITOX. Disponível em: <http://www.fiocruz.br/sinitox/2006/tab4_brasil.pdf>. Acesso em: 10 set. 2008.

CAPUANO Annalisa; MOTOLA Giulia; RUSSO Francesco; AVOLIO Amalia; FILIPPELLI Amelia; ROSSI Francesco; MAZZEO Filomena. Adverse drug events in two emergency departments in Naples, Italy: an observational study. Pharmacology Research, Londres, v. 50, n. 6, p. 631-636, 2004.

HERRERA CARRANZA, Joaquín. Aspectos éticos de la Atención Farmacéutica desde la perspectiva de la cadena terapéutica del medicamento. Pharmaceutical Care España, Barcelona, v. 3, n. 3, p. 223-226, 2001.

JOHNSON Jeffrey A.; BOOTMAN Lyle J. Drug-related morbidity and mortality: a cost-of-illness model. Archives of Internal Medicine, Chicago, v. 155, n. 18, p. 1949-1956, 1995.

LAZAROU Jason; POMERANZ Bruce H.; COREY, Paul. Incidence of adverse drug reactions in hospitalized patients - a meta-analysis of prospective studies. Journal of the American Medical Association, Chicago, v. 279, n. 15, p.1200-1205, 1998.

MARIN Nelly; LUIZA, Vera Lúcia; OSORIO-DE-CASTRO, Claudia G. Serpa; MACHADO-DOS-SANTOS, Silvio (Orgs.) Assistência farmacêutica para gerentes municipais. Rio de Janeiro: OPAS/OMS, 2003. 373 p. Disponível em: <http://www.opas.org.br/medicamentos/site/UploadArq/0080.pdf>. Acesso em: 17 set. 2008.

MENNITI-IPPOLITO Francesca; MAZZANTI Gabriela; SANTUCCIO Carmela; MORO Paola A; CALAPAI, Gioacchino; FIRENZUOLI Fabio; VALERI Andrea; RASCHETTI Roberto. Surveillance of suspected adverse reactions to natural health products in Italy. Pharmacoepidemiology and drug safety, Chichester, v. 17, n. 6, p. $626-635,2008$. 
MINISTÉRIO DA SAÚDE. CONSELHO NACIONAL DE SAÚDE. 1 a Conferência Nacional de Medicamentos e Assistência Farmacêutica. Relatório Final. 2005. 154p. Disponível em: <http://dtr2001.saude.gov.br/editora/produtos/livros/pdf/05_0069_M.pdf>. Acesso em: 10 set. 2008.

OLIVER Pascale; MONTASTRUC Jean-Louis. The nature of the scientific evidence leading to drug withdrawals for pharmacovigilance reasons in France. Pharmacoepidemiology and drug safety, Chichester, v. 15, n. 11, p.808812, 2006.

ORGANIZAÇÃO PAN-AMERICANA DA SAÚDE. Oficial de trabalho. Uso racional de medicamentos na perspectiva multiprofissional/Organização Pan-Americana da Saúde; Brasil. Ministério da Saúde; Agência Nacional de Vigilância Sanitária. Brasília: Organização Pan-Americana da Saúde, 2007. 28 p. Disponível em: <http://portal.saude.gov.br/portal/arquivos/pdf/ urm_rede_unida.pdf>. Acesso em: 12 set. 2008.

ORGANIZAÇÃO PAN-AMERICANA DA SAÚDE/ ORGANIZAÇÃO MUNDIAL DA SAÚDE/CONSELHO FEDERAL DE FARMÁCIA. O papel do farmacêutico no sistema de atenção à saúde. Brasília: Organização Mundial de Saúde/ Organização Pan-Americana da Saúde/Conselho Federal de Farmácia, 2004. 92 p. Disponível em: <http://www.opas.org.br/medicamentos/site/UploadArq/ HSE_PFS_BPF_1004.pdf>. Acesso em: 12 set. 2008.

WHA47.12 Role of the pharmacist in support of the who revised drug strategy. Pharmaceutical Care España, Barcelona, v. 2, n. 4, p. 282-284, 2000. Disponível em: <http://www.pharmaceutical-care.org/contenido.php? mod=archivobusqueda\& menu=3.2\&id=221\&tipo=completo $>$. Acesso em: 12 set. 2008.

WORLD HEALTH ORGANIZATION. Promoting rational use of medicines: core components. WHO Policy Perspectives on Medicines. n 5; Sept. 2002. 6 p. Disponível em: <http://www.who.int/medicines/publications/policyperspectives/ppm05en.pdf>. Acesso em: 10 set. 2008.

INTERNATIONAL PHARMACEUTICAL FEDERATION. Developing pharmacy practice: a focus on patient care, handbook 2006. Netherlands: World Health Organization/International Pharmaceutical Federation. 2006. 97p. Disponível em: <http://www.correofarmaceutico.com/documentos/ 041206WHO_PSM_PAR_2006.5.pdf>. Acesso em: 10 set. 2008. 